\title{
LAS ONG EN EL HORIZONTE DE LA MIGRACIÓN: DESAFÍOS EMERGENTES PARA LA CIENCIA JURÍDICA
}

\section{ONGS IN THE HORIZON OF MIGRATION: EMERGING GHALLENGES FOR JURIDICAL SCIENCES}

\author{
Francesco DE VANNA
}

\begin{abstract}
Resumen. La actividad de búsqueda y salvamento desarrollada por las ONG en el Mediterráneo cuestiona algunos postulados fundamentales de la teoría del derecho estatal: su carácter transnacional, el hecho de expresar a la sociedad civil (y no de la voluntad de un gobierno) y la conexión de sus actividades con las embarcaciones que recorren el mar impiden interpretar su condición jurídica a la luz de las consolidadas (y, en parte, vetustas) categorías jurídicas.

El derecho del mar, con sus normas, sus principios y sus convenciones, cuestiona los conceptos tradicionales de soberanía, jurisdicción y protección de derechos: de estos últimos, en particular, se espera una expansión que no se limite solamente al ciudadano, sino se extienda al hombre como tal.

Los Estados (ribereños), en particular los europeos, y la propia Europa respondieron a este desafío iniciando un proceso lento y sutil de criminalización de las ONG respaldado por investigaciones - que luego se revelaron sin fundamentos - de fiscales italianos. En esta perspectiva, el problema de la migración corre el riesgo de ser abordado solo en la dimensión «gerencial» propia de la seguridad y el control de fronteras.

La humanidad en viaje en el Mediterráneo, atravesando varias fronteras, revela el «escándalo» de la porosidad de las fronteras nacionales y revive la actualidad del ius migrandi que el Occidente liberal utilizó para la conquista del Nuevo Mundo.

Acusadas de actuar como un «taxi marítimo», las ONG revelan las paradojas políticas migratorias europeas: como fue oportunamente señalado, las ONG realizan una actividad político-humanitaria que controla y condiciona indirectamente el trabajo de los Estados en áreas extraterritoriales.
\end{abstract}

Palabras clave. ONG; Refugiados; Fronteras; Internacionalidad; Salvamento.

Abstract. Search and rescue activities carried out by NGOs in the Mediterranean Sea question fundamental principles of the state law theory: their transnational nature, the fact of being an expression of civil society (and not the will of governments), and the connection of their activities with vessels that sail at sea prevent one from interpreting their legal status in view of the consolidated (and, in part, very old) legal categories. 
The law of the sea - with its rules, its principles and its conventions - defies the traditional concepts of sovereignty, jurisdiction, and the protection of rights: of the latter, in particular, an increase is argued, not limited only to «citizens» but to humans as such.

The (coastal) States, in particular European ones, and Europe itself has replied to this challenge by initiating a slow and subtle process of criminalization of NGOs backed by investigations of Italian prosecutors without any legal fundament. In this regard, the problem of migration cannot be addressed only in a «managerial» dimension of security and border control.

Humanity traveling in the Mediterranean, crossing several borders, reveals the porosity of national borders and their "scandal", and revives the centrality of the ius migrandi as was used by the liberal West for the conquest of the New World. Accused of being «sea taxis», the NGOs reveal the paradoxes of the European migration policies: as it was properly observed, the NGOs carry out a political-humanitarian activity that controls and indirectly conditions the operations of the States in extra-territorial areas.

Keywords. NGO; Refugees; Borders; Internationality; Rescue

\section{EL SALVAMENTO EN EL MAR NO GUBERNAMENTAL}

La irrupción, ahora en forma estable, del fenómeno migratorio de masas en la agenda política europea ha puesto bajo apremio a gran parte de los países occidentales y a sus relativos sistemas jurídicos, sobre todo a las garantías constitucionales, que se revelaron frágiles y paradójicas.

En la figura del migrante, y específicamente en la del «solicitante de asilo», se reflejan las tensiones que atraviesan el constitucionalismo contemporáneo, detenido tras la defensa de la categoría tradicional de «ciudadano», asumida como la expresión por excelencia de la pertenencia de la mayoría a la comunidad política, y la promoción 
IUS ET SCIENTIA (ISSN: 2444-8478) 2019, Vol.5, nº 1, pp. 282-300

LAS ONG EN EL HORIZONTE DE LA MIGRACIÓN: DESAFÍOS EMERGENTES PARA LA CIENCIA JURÍDICA.

Francesco DE VANNA. Università degli Studi di Modena e Reggio Emilia, francesco.devanna@unimore.it

Recibido: 11/05/2019. Aceptado: 16/05/2019

DOI: http://dx.doi.org/10.12795/IETSCIENTIA.2019.i01.10

universal de los derechos humanos, más allá de cualquier límite, sea legal o sea territorial ${ }^{1}$.

Además emergen otros perfiles con la misma claridad: piénsese así en la disputa, no sólo política, llevada a cabo sobre el terreno de la reivindicación de «soberanía», de la cual los llamados movimientos populistas, en especial los reaccionarios, reivindican un «retorno» a lo nacional como la única instancia donde se pueden articular representación democrática y garantías sociales. Así se verifica, por tanto, una fuerte tensión entre el poder legislativo de los Estados vis-à-vis con las normas del derecho y de los convenios internacionales que, en general, en los sistemas legales europeos, asumen un rango jerárquico superior comparados con las fuentes internas en virtud del principio pacta sunt servanda ${ }^{2}$.

\footnotetext{
${ }^{1}$ El tema devino central para la reflexión jurídico-filosófica desde que, en la década de 1980, Michael Walzer inauguró, con Spheres of Justice, un intenso recorrido de estudios sobre la distribución de bienes dentro de sistemas «abiertos». Cfr. Th. Casadei, Il sovversivismo dell'immanenza: diritto, morale, politica in Michael Walzer, Milán, Giuffrè, 2012. Sobre paradojas y controversias en torno al concepto de ciudadanía se puede consultar, a modo de ejemplo, É. Balibar, Cittadinanza, Turín, Bollati Boringhieri, 2012. Cfr. también É. Balibar, S. Mezzadra, Ranabir Samaddar (eds.), The Borders of Justice, Filadelfia, Temple University Press, 2012. Además, para otras perspectivas, véase J. Habermas, Cittadinanza politica e identità nazionale (1991), en Morale, diritto, politica, Turín, Einaudi, 1992, pp. 105-138; D. Zolo (ed.), La cittadinanza. Appartenenza, identità, diritti, Roma-Bari, Laterza, 1994.

${ }^{2}$ El protocolo adicional a la Convención de las Naciones Unidas contra la delincuencia organizada transnacional para prevenir, suprimir y sancionar la trata de personas, en particular de mujeres y niños, firmado en Palermo en 2000, a menudo citado como base legal para acuerdos bilaterales, en particular con Libia, sin embargo, prevé la preeminencia de las normas internacionales que protegen los derechos humanos. Según el artículo 19 del protocolo: «Ninguna disposición de este protocolo afecta a los demás derechos, obligaciones y responsabilidades de los Estados e individuos derivados del derecho internacional, incluido el derecho internacional humanitario y el derecho internacional relacionados con los derechos humanos y, en particular, cuando sea aplicable, la convención de 1951 y el protocolo de 1967 sobre el estatuto de los refugiados y el principio de no devolución allí enunciados». Cfr. F. Vassallo Paleologo, Gli obblighi di soccorso in mare nel diritto sovranazionale e nell'ordinamento interno, en «Questione Giustizia», 2, 2018, pp. 215-224; K. Noussia, "The Rescue of Migrants and Refugees at Sea: Legal Rights and Obligations", en Ocean Yearbook, 2017, p. 155-170; M. Di Filippo, "Irregular Migration and Safeguard of Life at Sea: International Rules and Recent Developments in the Mediterranean Sea", en International Law of the Sea: Current Trends and Controversial Issues, editado por A. Del Vecchio, The Hague, Eleven, 2014, pp. 9-28; T. Scovazzi, "Human Rights and Immigration at
} 
IUS ET SCIENTIA (ISSN: 2444-8478) 2019, Vol.5, nº 1, pp. 282-300

LAS ONG EN EL HORIZONTE DE LA MIGRACIÓN: DESAFÍOS EMERGENTES PARA LA CIENCIA JURÍDICA.

Francesco DE VANNA. Università degli Studi di Modena e Reggio Emilia, francesco.devanna@unimore.it

Recibido: 11/05/2019. Aceptado: 16/05/2019

DOI: http://dx.doi.org/10.12795/IETSCIENTIA.2019.i01.10

El carácter irresuelto de estas tendencias favorece, cuanto menos indirectamente, el desarrollo de políticas públicas unilaterales y arbitrarias de los gobiernos nacionales, cada vez más comprometidos con una defensa a ultranza de las fronteras nacionales. Además también alienta el desarrollo de estilos comunicativos y propuestas legislativas comprometidas, en el mejor de los casos, con la «eficiencia» en la gestión de los flujos burocráticos y en los procedimientos administrativos (y no con la garantía del derecho) ${ }^{3}$, y más a menudo con la criminalización no sólo del «migrante», sino también de los actores comprometidos en su defensa ${ }^{4}$.

El resultado inmediato es el intento (exitoso, por cierto) de los Estados de sustraerse de la obligación - sancionada por la costumbre internacional, así como por numerosos instrumentos convencionales ${ }^{5}-$ de salvar vidas en el mar. Los Estados-nación derivan pues la responsabilidad en agencias supranacionales (como el caso de Frontex) o en la

Sea", en Human Rights and Immigration, editado por R. Rubio-Marín, Oxford, Oxford University Press, 2014, pp. $212-260$.

${ }^{3}$ Cfr. S. Penasa, L'approccio 'hotspot' nella gestione delle migrazioni: quando la forma (delle fonti) diviene sostanza (delle garanzie) efficientismo e garantismo delle recenti politiche migratorie in prospettiva multilivello, in Il diritto in migrazione. Studi sull'integrazione giuridica degli stranieri, editado por Fulvio Cortese, Gracy Pelacani, Nápoles, Editoriale Scientifica, 2017, pp. 396 - 427; D. Wilsher, Immigration Detention. Law, History, Politics, Cambridge, Cambridge University Press, 2012; F. Vassallo Paleologo, Diritti sotto sequestro. Dall'emergenza umanitaria allo stato di eccezione, Roma, Aracne, 2012; M. Agier, Managing the Undesirables. Refugee Camps and Humanitarian Governement, Londres, Polity Press, 2011. En la literatura especializada internacional cfr. L. Azoulai, K. De Vries, EU Migration Law: Legal Complexities and Political Rationales, Oxford, 2014; C. Costello, The Human Rights of Migrants and Refugees in European Law, Oxford, 2015; S. Peers, V. Moreno-Lax, M. Garlick, E. Guild, EU Immigration Law (Text and Commentary), II ed., vol. 3, Leiden-Boston, 2015; P. Boeles, M. Den Heijer, G. Lodder, K. Wouters (eds.), European Migration Law, Amberes, 2014; E. Guild, P. Minderhoud, The First Decade of EU Migration and Asylum Law, Leiden-Boston, 2011; S. Peers, EU Justice and Home Affairs Law, III ed., Oxford, 2012.

${ }^{4}$ Cfr. A. Pahladsingh, J. Waasdorp, Crimmigration law in the European Union, 2 vol., Oisterwijk, Wolf legal, 2016; M.A.H. Van Der Woude y P. Van Berlo, "Crimmigration at the Internal Borders of Europe? Examining the Schengen Governance Package", en Utrecht Law Review, 11, 2015, 61 ss., spec. 72. Respecto al contexto estadounidense consúltese: C. Cuauhtémoc García Hernández, Crimmigration Law, Chicago, American Bar Association, 2017.

${ }^{5}$ Según el artículo 98 de la Convención de las Naciones Unidas sobre el Derecho del Mar, firmada en Montego Bay en 1982, la Convención Internacional para la Seguridad de la Vida en el Mar (SOLAS) de 1974 y la Convenio Internacional sobre Búsqueda y Salvamento Marítimos (SAR). 
IUS ET SCIENTIA (ISSN: 2444-8478) 2019, Vol.5, nº 1, pp. 282-300

LAS ONG EN EL HORIZONTE DE LA MIGRACIÓN: DESAFÍOS EMERGENTES PARA LA CIENCIA JURÍDICA.

Francesco DE VANNA. Università degli Studi di Modena e Reggio Emilia, francesco.devanna@unimore.it

Recibido: 11/05/2019. Aceptado: 16/05/2019

DOI: http://dx.doi.org/10.12795/IETSCIENTIA.2019.i01.10

actividad espontánea de actores de la sociedad civil principalmente en las Organizaciones No Gubernamentales ${ }^{6}$.

El resultado en el mediano plazo, en cambio, es la intensificación de un proceso de laceración de las garantías previstas por los derechos constitucionales y las convenciones internacionales vulnerando el Estado de derecho y constriñendo al propio constitucionalismo a sufrir grandes contratiempos. Incluso induciendo a juristas y operadores a evocar objeciones y desobediencia a la ley considerada injusta ${ }^{7}$. Como estableció Thomas Casadei, describiendo el proceso en curso de negación de los derechos humanos fundamentales para muchos migrantes que conduce a condiciones de propia esclavitud real:

"Sujeción, sufrimiento, encarcelamiento - todo aquello que acompaña una condición de vulnerabilidad que deviene segregación - son las condiciones que caracterizan la esclavitud actual y los cuerpos recluidos (como si estuviesen encadenados), las 'vidas descartadas'. En los países occidentales, la clandestinidad - creada por la legislación migratoria - es el terreno sobre el cual crece toda crueldad, en detrimento del ius migrandi, del 'derecho a viajar', consagrado en documentos fundamentales a nivel internacional, pero también a expensas de las protecciones en el mundo del trabajo. En ese sentido, emerge la conexión, siempre más cruel, entre inmigración y esclavitud, entre trata de personas y esclavitud"».

\footnotetext{
${ }^{6}$ Cfr. A. Mitropoulos y B. Neilson, "Exceptional Times, Non-governmental Spacings, and Impolitical Movements", en Nongovernmental politics, editado por M. Feher, Zone Books, Nueva York, 2007, pp. 469-481.

${ }^{7}$ Más explícito sobre este punto es E. Santoro, I fondamenti del costituzionalismo alla prova del caso Diciotti: il sindacato sulle decisioni parlamentari e il punto di equilibrio fra poteri, in «Questione Giustizia», 14 de Marzo de 2019, disponible en línea en: http://www.questionegiustizia.it/articolo/ifondamenti-del-costituzionalismo-alla-prova-del- 14-03-2019.php.

${ }^{8}$ Th. Casadei, Corpi in transito: sulla tratta contemporanea, en La società degli individui, 3, 2018, pp. 140-154, p. 151. Del mismo autor se pueden consultar "Human wastes"? Contemporary forms of slavery and new abolitionism", en Soft power. Revista euro-americana de teoría e historia de la política, 2, 2016, pp. 109-124; "Modos y formas de la esclavitud contemporánea”, Derechos y Libertades, 39, 2018, pp. 35-
} 
La reivindicación de «soberanía», en el ámbito interno, es acompañada paralelamente por la deslocalización y desnacionalización del control fronterizo, cuya gestión se funda en un enfoque «gerencial» que responde a un imperativo de seguridad y administrativo. Así la cuestión de la migración se redujo a un mero control de fronteras en la perspectiva del orden público internacional, en una configuración despolitizada que dispone "en segundo plano y pospone el debate sobre las opciones políticas fundamentales que obligan a las personas a desplazarse de pasar de un país a otro, a recurrir a empresas de transporte no autorizadas de dudosa confiabilidad"9 como las organizaciones criminales que operan en Libia.

\section{LAS ONG EN LA TEORÍA DEL DERECHO}

Las Organizaciones No Gubernamentales se encuentran entre las figuras «noestatales» principales comprometidas en la gestión del fenómeno de la migración de $\operatorname{masas}^{10}$.

Para una mayor claridad, respecto de las Organizaciones No Gubernamentales es preferible ofrecer, incluso actualmente, una definición minimalista y negativa. Las organizaciones son evocadas habitualmente con un acrónimo (ONG) que describe primeramente aquello que no son, es decir, «no-gubernamental» implica que no

61. Cfr. también A. Dal Lago, Non-persone. L'esclusione dei migranti nella società globale, Milán, Feltrinelli, 2004.

${ }^{9}$ P. Cuttitta, "Ricerca e soccorso indipendenti nel canale di Sicilia", en InTrasformazione: Rivista di Storia delle Idee, p. 131. Cfr. también ID., "Borderizing the Island. Setting and Narratives of the Lampedusa Border Play”, en Acme: An International E-Journal for Critical Geographies, 13, 2014, pp. 196-219.

${ }^{10}$ Cfr. P. Alston, Non State Actors and Human Rights, Oxford, Oxford University Press, 2005. 
componen expresiones de gobiernos nacionales. Las ONG pertenecen a la formación del derecho internacional caracterizado por el surgimiento de formas de regulación jurídica espontánea y especialmente en carácter «privado». Por lo tanto, a diferencia de las organizaciones intergubernamentales, las ONG no son generadas por los Estados, sino por individuos.

En esta perspectiva, en el curso del siglo $\mathrm{XX}$, el crecimiento exponencial de estas organizaciones fue interpretada como la reacción de la sociedad civil a una concepción de la soberanía estatal que, en el campo de las relaciones internacionales y del derecho internacional, no reconocía como interlocutores válidos a los individuos ${ }^{11}$.

No obstante ello, las ONG persiguen fines públicos e intereses colectivos de solidaridad, como la tutela de los bienes comunes y del medio ambiente, la promoción de los derechos humanos, la protección de los consumidores, la lucha contra el tráfico de armas, y asumieron una responsabilidad institucional que legitima la participación en el proceso normativo transnacional. Es evidente, nuevamente a este respecto, que a pesar de la naturaleza autónoma, las ONG no son grupos colectivos autorreferenciales, o sea, organizados en torno a objetivos particulares que unen a sus adherentes. Por el contrario, se fijan objetivos institucionales altruistas, cuya proyección es extrínseca con respecto a los intereses inmediatos y las necesidades materiales de sus miembros.

Junto al carácter no-gubernamental, el rasgo que más típicamente caracteriza las Organizaciones No Gubernamentales consiste en la internacionalidad, pero en dos niveles. Es decir, desde el punto de vista de la extensión material de sus operaciones (transversal respecto de los límites territoriales de los Estados) y desde el punto de vista de su estructura organizativa (transnacional o, por así decirlo, «federal»). A este respecto,

\footnotetext{
${ }^{11}$ J.J. Lador-Lederer International non-Governmental Organizations and Economic entities: A Study in Autonomous Organization and Ius Gentium, AW Sythoff, Leiden, 1963, p. 27.
} 
vale precisar que, a pesar de la internacionalidad, las ONG se rigen por las leyes del Estado en el cual tienen su sede administrativa. Por tanto, a diferencia de las organizaciones intergubernamentales, las ONG se arraigan y operan en relación con la “cáscara de la soberanía estatal”,12, con la doble consecuencia que su estatus jurídico varía según el país en donde están arraigadas y que, cuando las estructuras organizativas de una ONG, se extienden por varios Estados, podría estar sujeta a diferentes regímenes legales.

No sorprende pues que, en la literatura especializada, se hayan referido al "no-status"13 precisamente para subrayar la «suspensión» en la cual queda relegada su condición jurídica en el horizonte internacional.

La ausencia de objetivos o propósitos lucrativos viene básicamente compartida y reconocida como fundamental en la condición de las ONG. En este sentido, se diferencian, a modo de ejemplo, de las empresas transnacionales. Ahora bien, la doctrina, sin embargo, no excluye que puedan perseguir y obtener ganancias, siempre y cuando estén destinadas al financiamiento de las actividades estatutarias y al pago de los empleados. En el tejido normativo del derecho italiano, las ONG se enmarcan, por consiguiente, en el campo de las organizaciones sin fines de lucro.

\section{LA REGULACIÓN DE LAS ACTIVIDADES DE SALVAMENTO EN EL MAR}

$\mathrm{Si}$ en el pasado reciente algunos estudiosos han tratado de enfatizar la función renovada de las ONG para fundar una transformación cualitativa en la concepción clásica

\footnotetext{
${ }^{12}$ Cfr. P. Benvenuti, "The Nature and Features of International Non-Governmental Organizations", en Italian Yearbook of International Law, 1978/79, pp. 84-102.

${ }^{13}$ Cfr. K. Martens, "Examining the (Non-) Status of Ngos in International Law", en Indiana Journal of Global Legal Studies, 10, 2, 2003, pp. 1-24.
} 
del derecho internacional - enunciando un "derecho global" auto-genético ${ }^{14}$-, las crónicas de los últimos meses testimonian la resistencia de los Estados y su continua centralidad en los procesos de gestión internacional de emergencias. Así todo ello con resultados siniestros en situaciones en donde la vida humana corre un riesgo directo e inmediato: tal es el caso de la emergencia derivada de la crisis de la gestión de los refugiados en la que Organizaciones No Gubernamentales jugaron - al menos hasta la promulgación, el 31 de julio de 2017, de la c.d. "Código de conducta relativo a las operaciones de rescate de migrantes en el mar" - un rol protagónico en el cumplimiento efectivo de obligaciones morales y legales específicas.

En 2014, la presencia de ONG en las aguas del Mediterráneo central se estructuró utilizando embarcaciones equipadas, contratadas con dicho fin y comprometidos en operaciones de búsqueda y rescate (search and rescue, SAR) que contribuyeron a salvar la vida de un número significativo de migrantes. De esta manera, las graves carencias o deficiencias de los Estados - en particular, va de suyo, en los litorales - con respecto a la obligación legal precisa de proporcionar ayuda en el mar (art. 98 de la UNCLOS $^{15}$ ) fueron parcialmente compensadas por la intervención voluntaria de estos actores «institucionales» de nuevo género "en cumplimiento de deberes morales antes que legales" ${ }^{16}$. Al asumir dicha tarea, las ONG transformaron las aguas internacionales

${ }^{14}$ Cfr. G. Ziccardi Capaldo, Diritto globale. Il nuovo diritto internazionale, Giuffrè, Milán, 2010; K. Nowrot, "Legal Consequences of Globalization: the Status of Non-Governmental Organizations under International Law", en Indiana Journal of Global Legal Studies, 2, 1999, pp. 579-645.

${ }^{15}$ El artículo 98 de la Convención de las Naciones Unidas sobre el Derecho del Mar, en particular, requiere que cada Estado obligue a los capitanes de las embarcaciones que enarbolan su propia bandera a establecer tres tipos de acciones que tengan como objetivo proteger las vidas de las personas en el mar. En primer lugar, el capitán debe brindar salvamento a cualquier persona que se encuentre y que pueda morir en el mar. En segundo lugar, debe proceder lo más rápidamente posible al rescate de las personas en peligro, en caso de tener alguna noticia. En tercer lugar, el capitán debe proporcionar asistencia después de una colisión entre embarcaciones. En los tres casos, se requiere que el capitán se conduzca acorde a lo dispuesto siempre y cuando no corran riesgo embarcación, tripulación y pasajeros.

${ }^{16}$ I. Papanicolopulu, "Immigrazione irregolare via mare, tutela della vita umana e organizzazioni non governative”, en Diritto, Immigrazione e Cittadinanza, 3, 2017, p. 2. El Estado italiano ofreció una 
IUS ET SCIENTIA (ISSN: 2444-8478) 2019, Vol.5, nº 1, pp. 282-300

LAS ONG EN EL HORIZONTE DE LA MIGRACIÓN: DESAFÍOS EMERGENTES PARA LA CIENCIA JURÍDICA.

Francesco DE VANNA. Università degli Studi di Modena e Reggio Emilia, francesco.devanna@unimore.it

Recibido: 11/05/2019. Aceptado: 16/05/2019

DOI: http://dx.doi.org/10.12795/IETSCIENTIA.2019.i01.10

"también en un teatro de una forma de contra-deslocalización: de una intervención político-humanitaria «no-gubernamental», es decir, que no sólo aumenta las capacidades de búsqueda y rescate localizadas de los actores gubernamentales, sino que también condiciona y controla las acciones de estos últimos en áreas extraterritoriales" ${ }^{\text {17. }}$.

Vale la pena mencionar que, de acuerdo con un principio general del derecho del mar, el Estado señalizado por la bandera izada en la embarcación ejerce plena jurisdicción sobre la misma, dondequiera que se encuentre ${ }^{18}$. Ello impide el ejercicio de la soberanía marítima total y exclusiva por parte de un Estado ribereño ya que, en una misma área marina y en una misma embarcación, pueden coexistir los poderes (reguladores y coercitivos) de una pluralidad de Estados y jurisdicciones (dependiendo de la porción de mar donde se encuentra la embarcación) ${ }^{19}$.

Así se deduce que, la misma suspensión que caracteriza el estatus jurídico-legal de las ONG en la sociedad internacional, se refleja ahora en la disciplina de las embarcaciones utilizadas en el Mediterráneo central en tanto rompen el tranquilizante binomio compuesto, por un lado, por embarcaciones comerciales (privados) y, por el otro, por

primera respuesta a la crisis humanitaria mediante la operación Mare Nostrum que duró en torno a un año, entre 2013 y 2014, antes de ser sustituida por la operación Triton lanzada por la Unión Europea, con un mandato humanitario sensiblemente limitado. Cfr. M. Tazzioli, "The Desultory Politics of Mobility and the Humanitarian-Military Border in the Mediterranean Mare Nostrum Beyond the Sea", en REMHU: Revista Interdisciplinar da Mobilidade Humana, 23, 2015, pp. 61-82.

${ }^{17}$ P. Cuttitta, Ricerca e soccorso indipendenti nel canale di Sicilia, cit., p. 139.

${ }^{18}$ I. Papanicolopulu, "Immigrazione irregolare via mare", cit., p. 5.

${ }^{19}$ «La posibilidad para otros Estados de ejercitar la propia jurisdicción sobre las embarcaciones que no izan la propia bandera depende de la zona marítima en la cual la embarcación se encuentra. Las aguas interiores son asimiladas al territorio terrestre del Estado y sobre ellas el Estado ribereño goza de plena soberanía. Los puertos forman parte de las aguas interiores y ello significa que el Estado puede impedir que embarcaciones extranjeras ingresen a sus puertos, incluso transportando personas rescatadas en el mar. Sin embargo, este derecho se encuentra con un límite en la norma que permite el ingreso a embarcaciones en caso de fuerza mayor o de peligro extremo. Más que un derecho de las embarcaciones, en estos casos se produce una cláusula de exclusión de responsabilidad, ya que la operación, que en otra circunstancia sería ilegal (el ingreso en el puerto sin autorización del Estado ribereño) deja de ser tal en presencia de una disposición absolutoria» (Ivi, p. 6). 
embarcaciones militares (estatales). Desde este punto de vista, por tanto, son sujetos híbridos.

El Código de Conducta, emanado a partir de la aplicación del Memorándum del 2 de Febrero de 2017 adoptado por el Gobierno italiano en el marco de la cooperación con las autoridades de Libia, fue diseñado y redactado para remediar y, en parte, evitar el marco regulatorio compuesto. En la imposibilidad de apelar a instrumentos jurídico-legales convencionales (o consuetudinarios) y para obtener una reducción significativa de los desembarcos, se decidió recurrir, con el consentimiento de la Unión Europea, a un instrumento de soft law no comparable a la ley y a los actos con fuerza de ley. Así se pidió entonces a las ONG que lo suscribieran, pero quedó claro inmediatamente su naturaleza jurídica era decididamente problemática ${ }^{20}$. Entre otras cosas, prevé la presencia de agentes de la policía judicial armados a bordo de las embarcaciones, la prohibición de entrar en aguas territoriales líbicas y la prohibición de efectuar transbordos.

El 11 de Agosto de 2017, las autoridades de Trípoli informaron a la Organización Marítima Internacional (OMI) del establecimiento de una zona de búsqueda y salvamento de Libia con una extensión de 70 millas de la costa inhibiendo aún todavía más la intervención directa de las diez ONG comprometidas hasta aquel momento. A partir de las amenazas realizadas por la «Guardia Costera» de Libia en distintas confrontaciones con las tripulaciones de las embarcaciones que participan en operaciones de salvamento, algunas ONG decidieron pues interrumpir sus actividades: Médecins Sans Frontières suspendió temporalmente sus actividades de búsqueda para luego ser sucedida, el 13 de

\footnotetext{
${ }^{20}$ Cfr. F. Mussi, "Sulla controversa natura giuridica del codice di condotta del Governo italiano relativo alle operazioni di salvataggio dei migranti in mare svolte da organizzazioni non governative", en Osservatorio sulle fonti, 3, 2017, pp. 1-10.
} 
IUS ET SCIENTIA (ISSN: 2444-8478) 2019, Vol.5, nº 1, pp. 282-300

LAS ONG EN EL HORIZONTE DE LA MIGRACIÓN: DESAFÍOS EMERGENTES PARA LA CIENCIA JURÍDICA.

Francesco DE VANNA. Università degli Studi di Modena e Reggio Emilia, francesco.devanna@unimore.it

Recibido: 11/05/2019. Aceptado: 16/05/2019

DOI: http://dx.doi.org/10.12795/IETSCIENTIA.2019.i01.10

Agosto de 2017, por Sea Eye y Save the Children y, finalmente, en Septiembre del mismo año, por MOAS (Migration Offshore Aid Station).

\section{LAS ONG ENTRE IUS MIGRANDI Y LA DOCTRINA DEL ESTADO}

La libertad de movimiento y circulación resultan del desarrollo contemporáneo del ius migrandi y, por tanto, tienen, en este sentido, una historia antigua. Su formulación teórica se remonta al siglo XVI, cuando el teólogo Francisco de Vitoria lo identificó como el fundamento del naciente derecho internacional como una concepción cosmopolita que nutría la amistad entre los pueblos ${ }^{21}$. La realidad, como es sabido, era bien distinta, ya que la migración de los indios en Occidente no sólo no era practicada, sino que ni siquiera era considerada pensable. Sin embargo, el desarrollo de este derecho desempeñó una función ideológica precisa legitimando las conquistas españolas en el Nuevo Mundo y, en los siglos siguientes, la cruel colonización de las grandes potencias europeas contra las poblaciones americanas, indias y africanas, siendo víctimas de trata y, no pocas veces, masacres reales.

${ }^{21}$ Luigi Ferrajoli subraya el carácter predominante del «derecho a migrar» como el principio consuetudinario del derecho internacional: «Toda la tradición liberal clásica siempre consideró al ius migrandi como un derecho fundamental. John Locke fundó allí la garantía del derecho a la supervivencia y la legitimidad misma del capitalismo: ya que el derecho a la vida, escribió, está garantizado por el trabajo, y todos pueden trabajar porque quieren, regresar al campo o emigrar a las "tierras no cultivadas de América", porque "existe suficiente tierra en el mundo para el doble de sus habitantes". Kant, a su vez, enunció aún más explícitamente no sólo el "derecho a emigrar", sino también el derecho a inmigrar que formuló como "el tercer artículo definitivo para la paz perpetua" identificado con el principio de una "hospitalidad universal". Y el artículo 4 del acta constitucional anexa a la Constitución francesa de 1793 estableció que "todo extranjero mayor de veintiún años que, domiciliado en Francia desde hace un año, que viva de su trabajo, o adquiera una propiedad, o se case con una ciudadana francesa, o adopte un niño, o mantenga a un anciano, es admitido en el ejercicio de los derechos del ciudadano".» (Cfr., ex multis, L. Ferrajoli, La questione migranti: Italia incivile, Europa incivile, en «Democrazia oggi», disponible en la siguiente dirección: http://www.democraziaoggi.it/?p=5742, pp. 3-4). 
Aunque a menudo es ignorada o incluso olvidada, incluso por los propios juristas, la Declaración Universal de los Derechos Humanos de 1948, en su artículo 13, reconoce explícitamente la libertad de circulación y el derecho a abandonar el propio país colocando pues un «deber ser» en los sistemas legales estatales (y supranacionales), llamados a adecuarse a la mencionada disposición a través de garantías legales y protecciones institucionales adecuadas.

En esta perspectiva, las ONG esbozan algunos desafíos decisivos a la teoría del derecho, de la cual exigen un aggiornamento a la luz de las relaciones transnacionales que se pueden establecer entre grupos articulados (y ordenamientos jurídicos) en diferentes territorios.

No obstante ello, en el caso de las ONG que trabajan en las aguas del Mediterráneo el desafío creció porque - insistiendo en las fronteras de los Estados - además de desmitificar el modelo de Estado-céntrico del derecho, ${ }^{22}$ revela el «escándalo» de la porosidad de las fronteras nacionales ("institución de instituciones" o "condición no democrática de la democracia"23). Como se observó en relación con el contexto europeo, también con respecto a la crisis del Sistema de Schengen:

«La reciente propensión de algunos gobiernos europeos a utilizar lo que resta de su poder para controlar las fronteras para limitar la afluencia de migrantes puede entenderse como un

\footnotetext{
${ }^{22}$ Consultar E. Santoro, "Confini”, in L. Barbari, F. De Vanna, Il "diritto al viaggio”. Abbecedario delle migrazioni, Turín, Giappichelli, 2018, pp. 31-36, quien observa: «El significado del límite se convierte en un campo de tensión entre las formas sociales que se identifican con un pedazo particular de territorio, es decir, los Estados, para los cuales un espacio definido es condición necesaria de existencia y principio ordenador del significado de la acción, y las formaciones supra-espaciales que, debido a su existencia y su significado interno, no tienen relación con el espacio delimitado, prescinden de la ubicación geográfica de sus elementos» (Ivi, p. 33).

${ }^{23}$ É. Balibar, “At the Borders of Citizenship: A Democracy in Traslation?", en European Journal of Social Theory, 13, 2010, pp. 315-322, p. 315.
} 
"regurgitación de soberanía": el estado-nación intenta resistir su declive, valorando su principal misión hobbesiana - proteger el orden interno y la seguridad de los ciudadanos - en el contexto, sin embargo, de una Europa continental en la cual (no el propio Estado, pero) el Estado como proyección de una comunidad nacional parece destinado al ocaso» ${ }^{24}$.

En años recientes, la acusación explícitamente dirigida a las ONG apunta a su supuesta connivencia con los traficantes de personas y promover la inmigración ilegal, utilizando las embarcaciones como «taxis marítimos». La insinuación, amplificada por el sistema de medios de comunicación en una propia criminalización y flaqueada por las investigaciones de procuradores sicilianos, resultó carente de evidencias investigativas apreciables. Por ello, las investigaciones fueron, hasta la fecha, en gran parte archivadas.

\section{BIBLIOGRAFÍA}

Agier, Michel, Managing the Undesirables. Refugee Camps and Humanitarian Governement, Londres, Polity Press, 2011.

Alston, Philip, Non State Actors and Human Rights, Oxford, Oxford University Press, 2005.

Anderson, Malcolm, Frontiers: Territory and State Formation in the Modern World, Cambridge, 1996

\footnotetext{
${ }^{24}$ M. Savino, "La crisi dei confini", en Rivista trimestrale di diritto pubblico, 3, 2016, p. 742. Cfr. además Ohmae, K., The Borderless World, Collins, Londres, 1990; M. Anderson, Frontiers: Territory and State Formation in the Modern World, Cambridge, 1996; Mezzadra, S., Neilson, B., Border as Method, or, the Multiplication of Labor, Duke University Press, Durham-Londres, 2013.
} 
Azoulai, Loïc, Karin De Vries, EU Migration Law: Legal Complexities and Political Rationales, Oxford, 2014.

Balibar, Étienne, “At the Borders of Citizenship: A Democracy in Traslation?", en European Journal of Social Theory, 13, 2010, pp. 315-323.

Balibar, Étienne, Cittadinanza, Turín, Bollati Boringhieri, 2012.

Balibar, Étienne, Sandro Mezzadra, Ranabir Samaddar (eds.), The Borders of Justice, Filadelfia, Temple University Press, 2012.

Benvenuti, Paolo, "The Nature and Features of International Non-Governmental Organizations", en Italian Yearbook of International Law, 1978/79, pp. 84-102.

Boeles, Pieter, Maarten Den Heijer, Gerrie Lodder, Kees Wouters (eds.), European Migration Law, Amberes, 2014.

Casadei, Thomas, Il sovversivismo dell'immanenza: diritto, morale, politica in Michael Walzer, Milán, Giuffrè, 2012.

Casadei, Thomas, “'Human wastes'? Contemporary forms of slavery and new abolitionism", en Soft power. Revista euro-americana de teoría e historia de la política, 2, 2016, pp. 109-124.

Casadei, Thomas, "Modos y formas de la esclavitud contemporánea", Derechos y Libertades, 39, 2018, pp. 35-61.

Casadei, Thomas. Corpi in transito: sulla tratta contemporanea, en La società degli individui, 3, 2018, pp. 140-154. 
Cuauhtémoc García Hernández, César, Crimmigration Law, Chicago, American Bar Association, 2017.

Costello, Cathryn, The Human Rights of Migrants and Refugees in European Law, Oxford, 2015.

Cuttitta, Paolo, "Ricerca e soccorso indipendenti nel canale di Sicilia", en InTrasformazione: Rivista di Storia delle Idee, 5, 2016, pp. 130-139.

Cuttitta, Paolo, "Borderizing the Island. Setting and Narratives of the Lampedusa Border Play", en Acme: An International E-Journal for Critical Geographies, 13, 2014, pp. 196-219.

Dal Lago, Alessandro, Non-persone. L'esclusione dei migranti nella società globale, Milán, Feltrinelli, 2004.

Di Filippo, Marcello, "Irregular Migration and Safeguard of Life at Sea: International Rules and Recent Developments in the Mediterranean Sea", en International Law of the Sea: Current Trends and Controversial Issues, editado por A. Del Vecchio, The Hague, Eleven, 2014, pp. 9-28.

Ferrajoli, Luigi, La questione migranti: Italia incivile, Europa incivile, en «Democrazia oggi», disponible en la siguiente dirección: http://www.democraziaoggi.it/?p=5742

Guild, Elspeth, Paul Minderhoud, The First Decade of EU Migration and Asylum Law, Leiden-Boston, Martinus Nijhoff Publishers, 2011.

Habermas, Jürgen, Cittadinanza politica e identità nazionale (1991), en Morale, diritto, politica, Turín, Einaudi, 1992, pp. 105-138. 
Lador-Lederer, J. Joseph, International non-Governmental Organizations and Economic entities: A Study in Autonomous Organization and Ius Gentium, AW Sythoff, Leiden, 1963.

Martens, Kerstin, "Examining the (Non-) Status of Ngos in International Law", en Indiana Journal of Global Legal Studies, 10, 2, 2003, pp. 1-24.

Mezzadra, Sandro, Neilson, Brett, Border as Method, or, the Multiplication of Labor, Duke University Press, Durham-Londres, 2013.

Mitropoulos, Angela, Brett Neilson, "Exceptional Times, Non-governmental Spacings, and Impolitical Movements", en Nongovernmental politics, editado por Michel Feher, Zone Books, Nueva York, 2007, pp. 469-481.

Mussi, Francesca, "Sulla controversa natura giuridica del codice di condotta del Governo italiano relativo alle operazioni di salvataggio dei migranti in mare svolte da organizzazioni non governative", en Osservatorio sulle fonti, 3, 2017, pp. 1-10.

Noussia, Kyriaki. "The Rescue of Migrants and Refugees at Sea: Legal Rights and Obligations", en Ocean Yearbook, 2017, p. 155-170.

Nowrot, Karsten, "Legal Consequences of Globalization: the Status of NonGovernmental Organizations under International Law", en Indiana Journal of Global Legal Studies, 2, 1999, pp. 579-645.

Ohmae, Kenichi, The Borderless World, Collins, Londres, 1990.

Papanicolopulu, Irini, "Immigrazione irregolare via mare, tutela della vita umana e organizzazioni non governative”, en Diritto, Immigrazione e Cittadinanza, 3, 2017. 
Peers, Steve, Violeta Moreno-Lax, Madeline Garlick, Elspeth Guild, EU Immigration Law (Text and Commentary), II ed., vol. 3, Leiden-Boston, 2015.

Peers, Steve, EU Justice and Home Affairs Law, III ed., Oxford, 2012.

Pahladsingh, Aniel, Jim Waasdorp, Crimmigration law in the European Union, 2 vol., Oisterwijk, Wolf legal, 2016.

Penasa, Simone. L'approccio 'hotspot' nella gestione delle migrazioni: quando la forma (delle fonti) diviene sostanza (delle garanzie) efficientismo e garantismo delle recenti politiche migratorie in prospettiva multilivello, in Il diritto in migrazione. Studi sull'integrazione giuridica degli stranieri, editado por Fulvio Cortese, Gracy Pelacani, Nápoles, Editoriale Scientifica, 2017, pp. 396 - 427.

Santoro, Emilio, “Confini”, in Luca Barbari, Francesco De Vanna, Il "diritto al viaggio”. Abbecedario delle migrazioni, Turín, Giappichelli, 2018, pp. 31-36.

Santoro, Emilio. I fondamenti del costituzionalismo alla prova del caso Diciotti: il sindacato sulle decisioni parlamentari e il punto di equilibrio fra poteri, in «Questione Giustizia», 14 de Marzo de 2019, disponible en línea en: http://www.questionegiustizia.it/articolo/i-fondamenti-del-costituzionalismo-allaprova-del- 14-03-2019.php.

Savino, Mario, "La crisi dei confini", en Rivista trimestrale di diritto pubblico, 3, 2016, pp. 739-759.

Scovazzi, Tullio. "Human Rights and Immigration at Sea", en Human Rights and Immigration, editado por R. Rubio-Marín, Oxford, Oxford University Press, 2014, pp. 212 -260. 
Tazzioli, Martina, "The Desultory Politics of Mobility and the Humanitarian-Military Border in the Mediterranean Mare Nostrum Beyond the Sea", en REMHU: Revista Interdisciplinar da Mobilidade Humana, 23, 2015, pp. 61-82.

Van Der Woude Maartje A.H. y Patrick Van Berlo, "Crimmigration at the Internal Borders of Europe? Examining the Schengen Governance Package", en Utrecht Law Review, 11, 2015, 61 ss.

Vassallo Paleologo, Fulvio, Gli obblighi di soccorso in mare nel diritto sovranazionale e nell'ordinamento interno, en «Questione Giustizia», 2, 2018, pp. 215-224.

Vassallo Paleologo, Fulvio, Diritti sotto sequestro. Dall'emergenza umanitaria allo stato di eccezione, Roma, Aracne, 2012.

Wilsher, Daniel. Immigration Detention. Law, History, Politics, Cambridge, Cambridge University Press, 2012.

Ziccardi Capaldo, Giuliana, Diritto globale. Il nuovo diritto internazionale, Giuffrè, Milán, 2010.

Zolo, Danilo (ed.), La cittadinanza. Appartenenza, identità, diritti, Roma-Bari, Laterza, 1994. 